\title{
Expert Opinions
}

\section{Can a new microscopy platform help to improve} clinical outcomes?

\author{
Sean R Koebley ${ }^{1} \&$ Jason Reed ${ }^{*, 1,2}$ \\ ${ }^{1}$ Department of Physics, Virginia Commonwealth University, Richmond, VA, USA; ${ }^{2}$ Massey Cancer Center, \\ Richmond, VA, USA
}

BioTechniques 65: 250-251 (November 2018) 10.2144/btn-2018-0102

Keywords: atomic force microscopy • DNA sequencing • genomics • nanotechnology

Next-generation sequencing (NGS), also referred to as 'second-generation sequencing', ushered in an era of plummeting sequencing costs that has enabled rapid progress in the research of genetic diversity, variants, transcription and epigenetics, among other fields. In the clinical sphere, NGS spurred the dream of precision medicine (PM) or personalized medicine, in which the genome of each patient (or pre-patient) is sequenced, and an optimal treatment is designed based on one's genetic code, lifestyle and environment [1]. Despite these gains and hopes, NGS is limited by its short-read approach and per-patient cost [2], and PM has yet to exhibit transformative reach, with only a small proportion of US patients currently receiving genome-informed therapy [3]. In order for PM to become a standard of care, there is an urgent need for fast, cheap and reliable genetic diagnostics to complement existing techniques. We offer a novel high-speed atomic force microscopy 'nanomapping' platform [4] to help meet the challenge.

NGS involves the sequencing and assembly of short reads (typically 100 bp), which limits its capacity to map elements spanning longer regions of the chromosome - including repeats, structural variants, and heterozygous variants that differ between the two parent chromosomes. Given that these elements account for the majority of diversity and pathogenetic variation between individuals [5], alternatives and modifications to NGS have been intensely explored. Single-molecule, 'third-generation' sequencers (e.g., PacBio and Oxford Nanopore) can produce reads of $10 \mathrm{~kb}$ or more, enabling the sequencing of previously inaccessible portions of the genome despite stochastic error rates of about 15\%. Between these third-gener- ation approaches, NGS improvements, and hybrid second- and third-generation assemblies, extremely accurate wholegenome or targeted sequencing efforts are now possible [2]. The effective translation of these techniques into a clinical setting is hindered by their time and cost, however: a whole-genome NGS run is, at best, currently priced at multiple thousands of US dollars, without considering computing or instrument costs, third-generation techniques are significantly more expensive, and both require several days and substantial computing power. These costs are undoubtedly justified in many cases, especially when a population-level genomic reference is being established [1], but cheaper, faster, targeted alternatives are surely preferable in many other scenarios. For example, if $\mathrm{PM}$ is to confront the common and daunting challenge of heterogeneous, evolving cancer [6], multiple rounds of rapid sequencing will surely be necessary to gather an updated diagnosis and gauge the efficacy of a given treatment, causing NGS or single-molecule sequencing costs to escalate rapidly.

To identify pathogenetic variants in a more targeted and efficient manner, genetic mapping offers a potential alternative to sequencing. At significantly lower cost and testing time, optical mapping techniques (e.g., Opgen and BioNano) can detect the locations of fluorescent probes along extended strands up to $250 \mathrm{~kb}$, and then compare the resulting map against a reference to determine the presence and character of indels, repeats or other variants [7]. Optical mapping has proved an important complement to sequencing that can essentially serve as a more detailed version of fluorescent in situ hybridization (FISH), but it suffers from limitations of its own: the template is only sparsely sampled, the nicking required to introduce fluorescent biomarkers can make heavily labeled portions prone to breakage, and most significantly, experimental and optical limitations typically render variants shorter than $10 \mathrm{~kb}$ inaccessible to mapping [2].

We recently showed that genetic mapping need not compromise resolution for speed and affordability [4]. Instead of measuring strand length and mapping fluorescent probes using optical techniques, which are realistically unable to resolve two probes closer than $\sim 1.5 \mathrm{~kb}$ or longer [7], we employ high-speed atomic force microscopy (HS-AFM) to image labeled DNA with effective resolution of $\sim 15 \mathrm{bp}$.

AFM was invented in the 1980s as a force sensing and imaging technique with resolution far surpassing that of optical microscopy [8]. The fundamental principle of AFM is similar to that of a record player: a sharpened tip detects topography as it is passed across a surface. The tip is attached to a flattened cantilever with a laser reflected off its rear face, such that a protrusion or depression encountered by the tip causes the cantilever to deflect and the laser's reflected angle to deviate. The laser deviation, and thus the tip height, can be measured with nanoscale accuracy. With AFM tips routinely manufactured by chemical etching to achieve diameters less than $5 \mathrm{~nm}$ and tip translation across the surface driven by piezoelectric stacks capable of subnanometer displacement, a three-dimensional map with nanoscale accuracy is collected when the tip is rastered across a surface. An AFM can operate at ambient temperature and pressure or in liquid, and has been successfully employed to investigate a range of biological processes [9]. However, 
the typical rate of AFM imaging - around 10 min of scanning required to image a $1-\mu \mathrm{m}^{2}$ area at $1 \mathrm{~nm} /$ pixel - has traditionally made its application to high-throughput diagnostics unfeasible.

Our HS-AFM, the fastest ever developed [10], achieves the same nanoscale resolution as a standard AFM at greater than 1000-times the rate of collection, affording tremendous statistical power and single-molecule sensitivity. Imaging a $1 \mu^{2}$ area with our system at $1 \mathrm{~nm} /$ pixel resolution is routinely achieved in $<1 \mathrm{~s}$. Strands of several kb can be measured in a single frame, and stitching together multiple frames enables molecules of virtually unlimited length to be read with an accuracy of $15 \mathrm{bp}$. Sample preparation is straightforward and flexible: the DNA is simply deposited onto a positively charged surface. The combination of imaging speed and accuracy allows for the lengths of several distinct species, e.g., indel or copynumber variants, to be determined with considerable statistical power.

Furthermore, we recently showed that endonuclease-inhibited Cas 9 can be employed as a programmable biomarker in HS-AFM imaging [4]. Since the diameter of the Cas9 molecule is greater than that of DNA, the location of bound Cas9 along a strand can be resolved, and we reported 95\% Cas9 binding accuracy under optimized conditions. Thus, in a manner similar to optical mapping but with vastly improved resolution, we can program one or more sgRNA-Cas9 complexes to bind a strategic set of targets, for example, the Alu repetitive element, to obtain a map of Cas 9 molecules for comparison with a reference. In addition, Cas9 labeling can be multiplexed to target other sites of interest, such as SNPs, for parallel variant assessment. With nanoscale resolution, the alignment of single-molecule maps becomes far more computationally straightforward than if labels are localized with multi-kb ambiguity, yielding reduced processing time and cost for assembling a consensus map [11]. Given its single-molecule sensitivity, 15 bp accuracy, and no amplification requirement, our method is amenable to small sample sizes - unlike single-molecule sequencing, which necessitates a clinically burdensome $\sim 10 \mu \mathrm{g}$ of DNA [2].

Beyond its improvement in mapping resolution, a major advantage of our technique is its accessibility. We have demonstrated that a commercially available optical DVD-pickup unit can serve effectively as the primary detection unit, an AFM tip costs less than US\$20, piezos and other instrument components are inexpensive, image processing and analysis can be conducted in parallel with scanning, computational assembly costs will be reduced due to improved accuracy, and sample preparation does not require specialized reagents. Compared with traditional sequencing, mapping, and even AFM platforms, our system is substantially more affordable we estimate the entire apparatus can be constructed for less than $\$ 10,000$, and individual samples can be prepared at negligible cost beyond that of standard reagents.

In summary, if the revolution in genomics research is to be translated into similarly transformative clinical outcomes, a mosaic of techniques will be necessary to confront the steepest challenges. Given that two major barriers to PM are the heterogeneity of genetic disease and the cost of sequencing [6], an approach with the capacity to accurately identify long-range variants at low cost and rapid run-time can complement the limitations of other approaches. Thus, in concert with NGS, single-molecule sequencing, and established assays, a nanomapping approach like HS-AFM scanning has the potential to significantly impact the clinical assessment of disease and diversity in the genomic age.

\section{Author contributions}

SRK and JR wrote and edited the article.

\section{Financial \& competing interests}

Funding for this work was provided by National Institutes of Health grants R01GM094388 and R01CA185189 to JR, by NIH IRACDA fellowship 5K12GM093857 to SRK, and in part, with funding from NCl Cancer Center Support Grant P30 CA016059 to the Massey Cancer Center. The authors have no other relevant affiliations or financial involvement with any organization or entity with a financial interest in or financial conflict with the subject matter or materials discussed in the manuscript apart from those disclosed.

No writing assistance was utilized in the production of this manuscript.

\section{Open access}

This work is licensed under the Attribution-NonCommercial-NoDerivatives 4.0 Unported License. To view a copy of this license, visit http://creativecommons.org/ licenses/by-nc-nd/4.0/

\section{References}

1. National Institutes of Health. All of Us - FAQs. (2018). https://allofus.nih.gov/about/programfaq

2. Sedlazeck FJ, Lee H, Darby CA, Schatz MC Piercing the dark matter: bioinformatics of longrange sequencing and mapping. Nat. Rev. Genet. 19(6), 329-346 (2018).

3. Marquart J, Chen EY, Prasad V. Estimation of the percentage of US patients with cancer who benefit from genome-driven oncology. JAMA Oncol. 4(8), 1093-1098 (2018).

4. Mikheikin A, Olsen A, Leslie $\mathrm{K}$ et al. DNA nanomapping using CRISPR-Cas9 as a programmable nanoparticle. Nat. Commun. 8(1), 1665 (2017).

5. Escaramís G, Docampo E, Rabionet R. A decade of structural variants: description, history and methods to detect structural variation. Brief. Funct. Genomics 14(5), 305-314 (2015).

6. Tannock IF, Hickman JA. Limits to personalized cancer medicine. N. Engl. J. Med. 375(13), 12891294 (2016).

7. Cao H, Hastie AR, Cao D et al. Rapid detection of structural variation in a human genome using nanochannel-based genome mapping technology. GigaScience 3(1), 34 (2014).

8. Binnig G, Quate CF, Gerber C. Atomic force microscope. Phys. Rev. Lett. 56(9), 930-933 (1986).

9. Dufréne YF, Ando T, Garcia R et al. Imaging modes of atomic force microscopy for application in molecular and cell biology. Nat. Nanotechnol. 12(4), 295-307 (2017).

10. Picco LM, Bozec L, Ulcinas A et al. Breaking the speed limit with atomic force microscopy. Nanotechnology 18(4), 044030 (2007).

11. Anantharaman TS, Mishra B, Schwartz DC Genomics via optical mapping. II: ordered restriction maps. J. Comput. Biol. 4, 91-118 (1997).

First draft submitted: 8 July 2018; Accepted for publication: 25 July 2018

Address correspondence to: Jason Reed, Department of Physics, Virginia Commonwealth University, Richmond, VA, USA; jcreed@vcu.edu

\section{To purchase reprints of this article contact:} s.cavana@future-science.com 